\section{Perfil Epidemiológico do Diabetes Mellitus Auto-Referido em Uma Zona Urbana de Juiz de Fora, Minas Gevais}

\begin{abstract}
RESUMO
Objetivo: Delinear um perfil epidemiológico do diabetes mellitus (DM) em uma zona urbana de Juiz de Fora usando os dados obtidos no cadastramento do Programa de Saúde da Família da Prefeitura Municipal de Juiz de Fora. Metodologia: Foram colhidas informações em 12.695 domicílios através da aplicação de um questionário baseado no Programa Avançado de Gerência em Atenção Primária a Saúde. A aplicação foi realizada por 180 entrevistadores acadêmicos da área de saúde. Os dados foram transferidos para o programa epidemiológico EPI-INFO versão 6.03. A análise do banco de dados referentes a população entre 30 e 69 anos permitiu a elaboração de um estudo sobre a prevalência do DM e sua relação com outros fatores. Resultados: A prevalência do DM auto-referido foi de $4,2 \%$ (861 pessoas) e variou de $0,9 \%$ (76) entre 30 e 39 anos a $11,6 \%$ (297) entre 60 e 69 anos. 17.1\% (147) relataram não tratar ou tratar irregularmente. Nos homens, a idade não interferiu no tratamento: nas mulheres, quanto menor a faixa etária, menor a adesão ao tratamento. O DM estava relacionado com outros agravos à saúde: cardiopatia $(r=4,8)$, hipertensão arterial sistêmica $(r=4,7)$, cegueira $(r=3,1)$ e incapacidade de locomoção $(r=3,9)$. Conclusão: A prevalência do DM auto-referido foi semelhante a de outros estudos nacionais. $O$ índice de diabéticos com tratamento irregular foi muito alto e relaciona-se com baixa renda e faixas etárias mais jovens no sexo feminino. O DM apresentou alta correlação com outros agravos à saúde: cardiopatia, hipertensão arterial sistêmica, cegueira e incapacidade de locomoção.
\end{abstract} (Arq Bras Endocrinol Metab 1999;43/3: 199-204).

Unitermos: Diabetes mellitus auto-referido; Epidemiologia; Diabetes mellitus; Doenças crônicas.

\begin{abstract}
Background: by using data from Juiz de Fora's Family Health Program survey, we aimed to assess the epidemiological profile of Diabetes Mellitus (DM) in an urban area in Juiz de Fora, MG. Brazil. Methodology: The source of information was a questionnaire, prepared according to the Primary Health Care Advancement Management Program, for the 12,695 homes visited. 180 students were selected from health courses to make the interviews, after having undergone a methodological training. Data obtained were organized using the software EPI-INFO 6.03 (WHO). Data bank analysis for the population between 30 and 69 years of age has led to the extensive study of DM prevalence and its association with other diseases. Results: Prevalence of self-reported DM was $4.2 \%(n=861)$ in general population, reaching $11.6 \%(n=297)$ of those between 60 and 69 years old and only $0.9 \%(n=76)$ among those between 30 and 39 years old. Irregular or no treatment was reported by 147 diabetics (17.1\%). Among men, age did not interfere with compliance; for women there was a correlation between age and adherence to treatment. Other health disturbances were significantly associated with DM: cardiopathy $(r=4.8)$, hypertension $(r=4.7)$, blindness $(r=3.1)$ and walking disabilities $(r=3.9)$. Conclusions: Self-reported DM prevalence was similar to other
\end{abstract}

\section{artigo original}

\author{
Alberto A. Larcher de \\ Almeida \\ Heloina L. M. Bonfante \\ Rodrigo de O. Moreira \\ Alberto K. Arbex \\ Gláucio S. de Souza \\ Leandro G. Maciel \\ Roger R. Godinbo \\ Giovanni Giannini
}

Núcleo de Pesquisa em Medicina Interna da Santa Casa do

Misericóndia de Juiz de Fora $e$ Universidade Federal de Juiz de Fora, Juiz de Fora, $M G$ 
national surveys. The high prevalence of non-adherence to treatment was significantly associated with low income and younger age in female gender. There was an important association of DM with other health problems, such as cardiopathy, hypertension, blindness and walking disabilities. (Arq Bras Endocrinol Metab 1999:43/3: 199-204).

Keywords: Self-reported diabetes mellitus; Epidemiology; Diabetes mellitus; Chronic diseases.

$\mathrm{N}^{\mathrm{s}}$ OS ÚITIMOS 30 ANOS, a melhora do saneamento básico e a prevenção de doenças infecto-contagiosas através de campanhas de vacinação e conscientização da população contribuiu para a redução da prevalência destas doenças, porém năo modificou o quadro de morbi-mortalidade por doenças crônicodegenerativas (1). Dessas, o diabetes mellitus (DM) assume evidente importância no Brasil, pois acomete $7,6 \%$ dos indivíduos entre 30 c 69 anos $(2,3)$, com prevalência crescente particularmente no segmento urbanizado da população. Estes dados foram obtidos quando prevalecia o critério da OMS que considerava diabético o indivíduo com glicose plasmática de jejum $\geq$ $140 \mathrm{mg} / \mathrm{dL}$. O crescimento da prevalência é um fenômeno mundial e nos Estados Unidos um estudo com 18.825 adultos com $\geq 20$ anos de idade, mostrou a prevalência de $5,1 \%$ de DM diagnosticado por médico com $2,7 \%$ de DM não diagnosticado (4). O DM cursa com complicações cardiovasculares, neurológicas, renais e oftamológicas e cstá entre as 10 maiores causas de morbi-mortalidade no Brasil (5), acarretando altos custos ao sistema de saúde $(6,7)$. A mortalidade proporcional pelo DM em Juiz de Fora foi de $3,6 \%$ no período de 1981 a 1990 (8). O tratamento correto diminui a morbi-mortalidade e melhora a qualidade de vida, além de diminuir os custos com as complicações $(9,10)$.

A cidade de Juiz de Fora, localizada na Zona da Mata de Minas Gerais, é um centro regional de referência política e econômica nos setores secundário c terciário. Possui em torno de 420.000 habitantes distribuídos em quatro regiões: norte, sul, leste c oeste. Para identificar possíveis fatores de risco em áreas de baixo nível sócio-econômico, a Prefeitura Municipal de Juiz de Fora (PMJF) decidiu, através do Programa de Saúde da Família (PSF) e com o apoio da Universidade Federal de Juiz de Fora (UFJF), realizar um cadastramento nestas áreas. O PSF ć composto por 39 equipes que atendem 1.000 famílias cada uma (abrangendo cerca de $30 \%$ da população da cidade) c o cadastramento foi realizado tanto em áreas já cobertas por esse programa (zona leste) como $\mathrm{cm}$ áreas onde se planeja implantá-lo (zonas norte e oeste).
O objetivo deste estudo foi delinear um perfil epidemiológico do DM na região bana de Juiz de Fora, usando como base os dados do cidastramento do PSF, de modo que açóes específicas educativas c preventivas possam ser planejadas c aplicadas por cquipes de saúde voltadas à atenção primária. Dentre as infor maçoes, foi detectada a prevalência auto-referida do DM na população, baseada no diagnóstico feito por médico e informado pelo paciente, que também referia a presença de outros agravos crônicos à saúde. Buscouse a identificação dos aspectos sociais e econômicos dos diabéticos referidos $c$ informaçóes sobre a adesão ao tratamento preconizado pelos serviços de saúde.

\section{METODOLOGIA}

Foram utilizados os dados obtidos pelo Programa de Saúde da Família da PMJF em conjunto com a UFJF. O cadastramento foi realizado prioritariamente nas zonas leste, oeste e norte devido às suas características de árcas urbanas com significativa carência sócio-econômica cà limitação de recursos. Na zona leste, com o objetivo de melhorar as ações de saúde das equipes já formadas e nas zonas norte e oeste, a fim de fornecer um perfil geral da população para futura implantação do programa.

Para a colcta de dados foi utilizado um questionário, aplicado entre maio de 1996 e dezembro de 1997, preparado segundo o Programa Avançado de Gerência cm Atenção Primária à Saúde da Universidade Aga-Klan e obtido do Plano Integrado de Educação c Saúde, projeto da UFJF voltado à melhoria do sistema de saúde da região. A l’ parte do questionário cra referente a dados individuais de cada morador, incluindo idade, sexo e situação trabalhista. A 2 parte se referia a questões de moradia, higiênico-sanitárias e econômicas (renda familiar). A 3 a parte consistia de perguntas sobre a prevalência de doenças crônicas entre os moradores, incluindo o DM, cardiopatia, hipertensão arterial sistêmica, cegueira e incapacidade de locomoção, entre outras. Estes diagnósticos foram feitos por médicos e referidos pelas pessoas entrevistadas.

A aplicação deste questionário foi realizada por 180 entrevistadores, todos acadêmicos da área de saúde (medicina e enfermagem), que receberam trcinamento prévio teórico e prático, através de teste em árca piloto. Cada residência era visitada no máximo 3 vezes e se a responsável pela casa (a quem o questionário era aplicado) se recusava a responder ou não fosse encontrada, o serviço social do posto local de saúde era avisado e os dados recolhidos posteriormente. $20 \%$ das residências visitadas se negaram a receber os entrevistadores. 
Foram colhidas informações $\mathrm{cm} 12.695$ domicílios pertinentes a 50.826 pessoas. Os dados foram transferidos para o programa epidemiológico EPI-INFO versão 6.0. A análise dos dados permitiu a claboração de um estudo epidemiológico sobre o DM. Foram destacados e analisados os dados pertinentes à parcela da população entre 30 e 69 anos de idade, visando comparação com a literatura. Avaliou-se o tratamento do DM informado, considerando-se tratamento regular a adesão às prescrições do médico e irregular o relato de "não tratar" ou "tratar irregularmente". A prevalência de outras doenças crônicas foi avaliada na população diabética c na não diabética. Para comparação entre grupos, quando as variáveis não apresentavam distribuição normal, utilizamos o teste de Kruskall-Wallis. Nas demais comparações, foi utilizado o teste " $t$ " de Student. O limite de significância foi de $5 \%$ e o valor para intervalo de confiança foi de $95 \%$.

\section{RESULTADOS}

Os dados referentes a prevalência do DM na população investigada encontram-se na tabela 1 . Foram cstudados especificamente os 20.287 indivíduos entrc 30 e 69 anos. Destes, $861(4,2 \%)$ declararam ser portadores de DM, sendo $591(68,6 \%)$ do sexo feminino e 270 $(31,4 \%)$ do masculino $(r=1,89$ e $\mathrm{p}<0,001)$. A prevalência do DM foi de $0,9 \%(76)$ na faixa etária entre $30 \mathrm{c}$ 39 anos, 3,8\% (230) entre 40 e 49 anos, 6,9\% (258) entre 50 e 59 anos e $11,6 \%(297)$ entre 60 c 69 anos.

Quanto a adesão ao tratamento, $714(82,9 \%)$ relataram tratar regularmente o DM enquanto que 147 $(17,1 \%)$ relataram não tratar ou tratar irregularmente. Embora não exista associação entre sexo e adesão ao tratamento $(r=0,90$ e $p=0,57)$, o tratamento irregular do DM mostrou-se influenciado pelas diferentes faixas ctárias nas mulheres $(p=0,01)$, o mesmo năo ocorrendo com os homens $(p=0,8)$ e tabela 2 . O fator cscolaridade foi estudado e não mostrou relação estatistica- mente significante com o tratamento. A figura 1 reflete a relação entre baixa renda familiar e o tratamento irregular do $\mathrm{DM}(\mathrm{p}=0,007)$.

Dos 861 diabéticos, $448(52 \%)$ relataram ser portadores também de hipertensão arterial $(r=4,7$; $\mathrm{p}<0,001) ; 176(20,4 \%)$ relataram ser cardiopatas $(\mathrm{r}=4,8 ; \mathrm{p}<0,001) ; 18(2,1 \%)$ relataram cegueira $(\mathrm{r}=3, \mathrm{l}$; $\mathrm{p}<0,00 \mathrm{l})$ e $32(3,7 \%)$ possúam incapacidade de locomoção $(r=3,9 ; p<0,001)$. A prevalência destes agravos nas pessoas sem DM referido foi 3 a 4 vezes menor. As demais doenças crônicas questionadas (doenças pulmonares, mentais e renais) não mostraram correlação estatisticamente significante com o DM. A tabela 3 mostra os dados referentes a prevalência de doenças crônicas referidas na população entre 30 e 69 anos e a sua associação com o DM, numa correlação altamente significante $(\mathrm{p}<0,001)$.

\section{DISCUSSĀO}

A população das zonas leste, oeste c norte de Juiz de Fora tem distribuição homogênea, quando avaliada segundo o sexo ( $1: 1)$. Entretanto, é uma zona de baixo nível educacional, onde grande parte da população $(68,6 \%)$ tem, no máximo, o $I^{\circ}$ grau completo. A renda familiar média é muito baixa, estando por volta dos 4,4 salários mínimos $(\mathrm{sm})$. A área conta com uma boa infraestrutura sanitária, que contribui para menor prevalência de doenças infecciosas e maior de doenças crônicodegenerativas (dados obtidos no cadastramento).

$\mathrm{Na}$ população estudada foi encontrada a prevalência de DM referido de $2,2 \%$ na população total (Tabela 1) e de 4,2\% na população entre 30 e 69 anos, coincidindo com estudos randomizados no cstado do Rio de Janeiro (3) e também com os dados do Grupo de Estudo da Prevalência de Diabetes Mellitus no Brasil (2), que em 1992 apontou uma média de prevalência em torno de $7,6 \%$ no território nacional, sendo apenas $3,8 \%$ já diagnosticados. Nos Estados

Tabela 1 - Prevalência do diabetes mellitus auto-referido numa zona urbana de Juiz de Fora

\begin{tabular}{|c|c|c|c|c|c|c|}
\hline \multirow{2}{*}{$\begin{array}{l}\text { Sexo } \\
\begin{array}{l}\text { Faixa Etária } \\
\text { (anos) }\end{array}\end{array}$} & \multicolumn{3}{|c|}{ Feminino } & \multicolumn{3}{|c|}{ Masculino } \\
\hline & População & Diabéticos & Prevalência & População & Diabéticos & Prevalência \\
\hline $\begin{array}{l}<29 \\
30-39 \\
40-49 \\
50-59 \\
60-69 \\
>70\end{array}$ & $\begin{array}{l}14.159 \\
4.233 \\
3.312 \\
2.013 \\
1.457 \\
1.320\end{array}$ & $\begin{array}{c}37 \\
47 \\
137 \\
184 \\
223 \\
140\end{array}$ & $\begin{array}{l}0,3 \% \\
1,1 \% \\
4,1 \% \\
9,1 \% \\
15,3 \% \\
10,6 \%\end{array}$ & $\begin{array}{r}14.296 \\
3.662 \\
2.779 \\
1.721 \\
1.707 \\
767\end{array}$ & $\begin{array}{l}25 \\
29 \\
93 \\
74 \\
74 \\
50\end{array}$ & $\begin{array}{l}0,2 \% \\
0,8 \% \\
3,3 \% \\
4,3 \% \\
6,7 \% \\
6,5 \%\end{array}$ \\
\hline Total & 26.494 & 768 & $2,9 \%$ & 24.332 & 345 & $1,4 \%$ \\
\hline
\end{tabular}


Tabela 2 - Tratamento regular e irregular em diabéticos auto-referidos segundo o sexo feminino $(n=591)$ ou masculino $(n=270)$ e a faixa etária.

\begin{tabular}{|c|c|c|c|c|}
\hline Sexo & \multicolumn{2}{|c|}{ Feminino } & \multicolumn{2}{|c|}{ Masculino } \\
\hline \multirow{2}{*}{$\begin{array}{l}\text { Faixa Etária } \\
\text { (anos) }\end{array}$} & \multicolumn{2}{|c|}{ Tratamento } & \multicolumn{2}{|c|}{ Tratamento } \\
\hline & Regular & Irregular & Regular & Irregular \\
\hline $\begin{array}{l}30 \text { a } 39 \\
40 \text { a } 49 \\
50 \text { a } 59 \\
60 \text { a } 69\end{array}$ & $\begin{array}{r}36(76,6 \%) \\
104(75,9 \%) \\
162(88,0 \%) \\
191(86,4 \%)\end{array}$ & $\begin{array}{l}11(23,4 \%) \\
33(24,1 \%) \\
22(12,0 \%) \\
32(13,6 \%)\end{array}$ & $\begin{array}{l}23(79,3 \%) \\
74(79,6 \%) \\
61(82,4 \%) \\
63(85,1 \%)\end{array}$ & $\begin{array}{l}6(20,7 \%) \\
19(20,4 \%) \\
13(17,6 \%) \\
11(14,9 \%)\end{array}$ \\
\hline Total & $493(83,4 \%)$ & $98(16,6 \%)$ & $221(81,8 \%)$ & $49(18,2 \%)$ \\
\hline
\end{tabular}

Tabela 3 - Prevalência de outros agravos à saúde em diabéticos e não diabéticos auto-referidos em Juiz de Fora.

\begin{tabular}{lcccc}
\hline & Hipertensão & Cardiopatia & $\begin{array}{r}\text { Incapacidade } \\
\text { de Locomoção }\end{array}$ & Cegueira \\
Diabéticos & $448(52,0 \%)$ & $176(20,4 \%)$ & $32(3,7 \%)$ & $18(2,1 \%)$ \\
Năo Diabéticos & $3603(18,5 \%)$ & $977(5,0 \%)$ & $189(1,0 \%)$ & $133(0,7 \%)$ \\
Total & $4051(20,0 \%)$ & $1153(5,7 \%)$ & $221(1,1 \%)$ & $151(0,7 \%)$ \\
\hline
\end{tabular}

* $p<0.001$ para todas as doenças

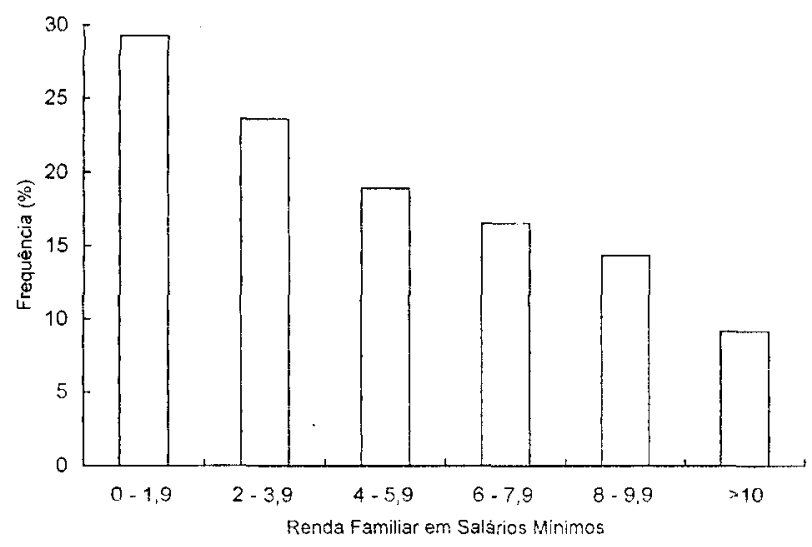

Figura 1. Relaçâo entre renda familiar e tratamento irregular dos diabéticos auto-referidos.

Unidos, Harris et al (4) discutem os dados do estudo NHANES III, realizado de 1988-1994 que apontou $5,1 \%$ de DM diagnosticado por médico em adultos com 20 anos ou mais c o encontro de $2,7 \%$ de DM não diagnosticado, taxa menor que a brasilcira porém ainda muito elevada, tendo em vista as complicaçōes que o diabetes acarreta $(10,11)$. O maior número de mulheres diabéticas $\mathrm{em}$ relação aos homens $(\mathrm{r}=1,89$; $\mathrm{p}<0,001)$ pode ter se originado do método utilizado, onde se preferiu entrevistar a "dona da casa", da maior freqüência com que as mulheres visitam os postos de saúde, dos fatores culturais e da maior prevalência da obesidade no sexo feminino.
Os fatores genéticos não foram pesquisados co nível sócio econômico é baixo, o que nos trabalhos americanos se correlaciona com aumento da prevalência do DM (12).

Quanto ao alto índice de não adesão ao tratamento do $\mathrm{DM}(17,1 \%)$, os motivos são semelhantes aos de outras patologias crônicas e entre eles estão: (i) desconhecimento pelo diabético que um ótimo controle glicêmico pode reduzir e retardar as complicações (13); (ii) custo do tratamento; (iii) dificuldade em adquirir a medicação; (iv) preconceito em utilizar medicamentos de forma crônica; (v) uso de insulina scr considerado um incômodo pclo paciente; (vi) dificuldades em modificar o estilo de vida, começando pelos hábitos alimentares.

Dentre os fatores relacionados à aderência ao tratamento, sexo e idade mostraram-se muito importantes (Tabela 2). Enquanto que nos homens não existe diferença significante na adesão ao tratamento nas diferentes faixas etárias $(p=0,8)$, nas mulheres este índice apresenta-se variável $(p=0,01)$. $O$ alto número de mulheres que tratam irregularmente a doença nas faixas etárias mais jovens (24,1\% entre 40 e 49 anos) é devido principalmente a ausência de complicações. Seguindo a historia natural da doença, nas faixas mais avançadas há o aparccimento das complicações crônicas (além de outras situações decorrentes da idade) fazendo com que a procura aos serviços de saúde seja maior (14) e melhore a aderência às orientações dos médicos (12\% das mulheres entre os 50 e 59 anos tratam irre- 
gularmente; $r=2,34$ c $p=0,006$ ). Paralelamente, a dificuldade desses serviços em alertar os pacientes precocemente sobre sua doença faz com que a adesão total ao tratamento só seja feita quando a mesma encontra-se sintomática e, muitas vezes, complicada.

Os dados da figura l mostram a relação entre o tratamento informado e a renda familiar $(p=0,007)$. $\mathrm{O}$ número de pessoas que tratam irregularmente a doença é muito maior quando a renda familiar é baixa, possivelmente por impedir a alimentação correta e a aquisição dos medicamentos, além de se associar, na maioria das vezes, a um baixo nível educacional. A medida que a renda familiar aumenta há diminuição no número de pessoas que tratam irregularmente, o que se confirma quando se comparam os diabéticos com renda familiar de 0 a $1,9 \mathrm{sm}$ com os de renda igual ou superior a $10 \mathrm{sm}$, mostrando íntima relação entre a situação econômica e a aderência ao tratamento do DM.

A tabela 3 mostra a forte relação do DM com outros agravos à saúde como hipertensão arterial sistêmica (HAS), cardiopatias, incapacidade de locomoção e cegueira. A alta correlação entre HAS $(r=4,7)$ e DM já é bem conhecida e se explica inclusive pela alta prevalência da síndrome plurimetabólica (HAS, resistência a insulina, obesidade e dislipidemia), que freqüentemente precede o DM tipo 2 (15). A HAS potencializa os efeitos deletérios do descontrole glicêmico crônico sobre a microvasculatura (16). O alto risco $(\mathrm{r}=4,8)$ relacionado à cardiopatia pode ser explicado pela presença de hipertensão e pelo desenvolvimento de doença aterosclerótica (17). O risco de cegueira $\mathrm{em}$ pacientes diabéticos foi muito significante $(r=3,1)$ e estes dados condizem com o fato de que o DM é a principal causa de cegueira no mundo, podendo ser justificado pelo importante acometimento microvascular do diabético (18). Em relação à incapacidade de locomoção, a sua prevalência também foi maior no diabético $(r=3,9)$. Este fato pode ser explicado pela evolução natural da doença, que cursa com macroangiopatia, microangiopatia e neuropatia.

\section{CONCLUSÕES}

A prevalência do DM auto-referido de $4,2 \%$ na população entre 30 c 69 anos em uma zona urbana da cidade de Juiz de Fora é semelhante à dos estudos nacionais, caracterizando também maior prevalência no sexo feminino.

O número de pacientes com tratamento irregular foi alto e se relaciona com baixa renda e faixa ctária mais jovem no sexo feminino.
O DM apresentou alta correlação com outros agravos à saúde, como HAS, cardiopatias, cegueira e incapacidade de locomoção.

Os resultados poderão ser utilizados para aperfeiçoar as ações de saúde dos diabéticos nos centros de atenção primária da comunidade.

\section{AGRADECIMENTOS}

Dr. Márcio José Alves pela elaboração do questionário e pela ajuda na análise estatística dos dados e Dr. Celso de Castro Matias Neto pelo apoio e pela abertura à participação dos estudantes de medicina no Projeto de Saúde da Família da Secretaria de Saúde da PMJF.

\section{REFERÊNCIAS}

1. Lessa 1, Mendonça GA. Teixeira MT. Non-communicable chronic diseases in Brazil: from risk factors to social impact. Bol Oficina Sanit Panam 1996; 120:389-413.

2. Malerbi DA, Franco LJ. Multicenter study of the prevalence of diabetes mellitus and impaired glucose tolerance in the urban brazilian population aged 30-69 yr The Brazilian Cooperative Group on the Study of Diabetes Prevalence. Diabetes Care 1992; 15:1509-16.

3. Oliveira JE, Milech A, Franco LJ. The prevalence of dia betes in Rio de Janeiro. Brazil. The Cooperative Group for the Study of Diabetes Prevalence in Rio de Janeiro. Diabetes Care 1996; 19:663-6.

4. Harris MI, Flegal KM, Cowie CC, Eberhardt MS, Goldstein et al. Prevalence of diabetes, impaired fasting glucose and impaired glucose tolerance in US adults (The Third National Health and Nutrition Examination Survey, 19881994). Diabeles Care 1998;21:518-24.

5. Laurenti $R$, Fonseca $L A$, da Costa Júnior ML. Diabetes mellitus mortality in the municipality of São Paulo (Brazil). Evolution over a period of 79 years (1900-1978) and analysis of some aspects of the association of causes. Rev Saude Publ 1982;16:77-91.

6. Hart WM, Espinosa C. Rovira J. Costs of known diabetes mellitus in Spain. Med Clin (BarC) 1997; 109:289-93.

7. Jacobs J, Sena M, Fox N. The cost of hospitalization for the late complications of diabetes in the United States. Diab Med 1991;8(supl 2):3-9.

8. Marques $\mathrm{MH}$, Costa MB, Ibiapina EC, Ibiapina CC Almeida RM, Almeida AAL. Mortalidade na população diabética adulta em Juiz de Fora, MG. VIII Congresso Brasileiro de Diabetes, Fortaleza. CE. Arq Bras Endocrinol Metab 1991;35(supl 2):10.

9. Santiago JV. Intensive management of insulin dependent diabetes: risks, benefits and unanswered questions. J Clin Endocrinol Metab 1992;75:977.

10. Harris MI. Diabetes in America: Epidemiology and scope of the problem. Diabetes Care 1998:21(supl 3):11-4.

11. Eastman RC, Cowie CC, Harris Ml: Undiagnosed diabetes or impaired glucose tolerance and cardiovascular risk. Diabetes Care 1997:20:127-8. 
12. Haffner SM. Epidemiology of type 2 diabetes: risk factors. Diabetes Care 1998;2l (supl 3):3-6.

13.Diabetes Control and Complications Trial Research Group: The effect of intensive treatment of diabetes on the development and progression of long-term complications of insulin-dependent diabetes mellitus. N Engl J Med 1993:329:977-86.

14. Mustard CA, Kaufert P, Kozyrskyj A, Mayer T. Sex differences in the use of health care services. $N$ Engl J Med 1998:339:1678-83.

15. American Diabetes Association: Treatment of Hypertension in Diabetes (Consensus Statement). Diabetes Care 1996; 19(supl 1):S107-S113.

16. Schwartz CJ, Valente AJ, Sprague EA, Kelley JL, Cayatte AJ. Rozek MM. Pathogenesis of the atherosclerotic lesion. Implications for diabetes mellitus. Diabetes Care 1992; 15:1156-67.

17. Pyorala K, Laakso M. Uusitupa M. Diabetes and atherosclerosis. An epidemiological view. Diabetes Metab Rev 1987;3:463-528.
18. Pfeiffer A, Schatz H. Diabetic microvascular complications and growth factors. Exp Clin Endocrinol Diabetes $1995 ; 103: 7-14$.

\section{Endereço para correspondência:}

Alberto A. Larcher de Almeida

Centro de Estudos da Santa Casa de Misericórdia - Núcleo de Pesquisa em Medicina Interna

Santa Casa de Misericórdia de Juiz de Fora

Av. Barõo do Rio Branco, 3353

36021-630, Juiz de Fora, $M G$

e-mail: santacasa-jf@fusoes.com.br 